\title{
EP-38
}

\section{Hepatic resection for hepaticolithiasis: A single-centre experience of 8 cases}

\author{
Prasad PANDE ${ }^{*, 1}$, Gunjan DESAl', Prasad WAGLE ${ }^{2}$
}

'Department of Surgical Gastroenterology, MGM New Bombay Hospital, Vashi, Navi Mumbai, India

²Department of Surgical Gastroenterology, Lilavati Hospital \& Research Centre, Mumbai, India

Introduction: Hepaticolithiasis is frequently not amenable to endotherapy, and surgery forms the mainstay of treatment. This study highlights the outcomes of major liver resection for this benign pathology.

Methods: Our tertiary hepatobiliary centre performed 8 liver resections for hepaticolithiasis between January 2010 and December 2019. Demographic data, clinicoradiological data, surgical details, and outcomes were recorded.

Results: Out of 8 patients, 6 were females. Median age was 54 years. 6 patients underwent left hepatectomy, and 1 each underwent right hepatectomy and right trisectionectomy. Trisectionectomy was done after liver volumetry showed a future liver remnant volume of $48 \%$ with right posterior section atrophy. Mean intra-operative blood loss was $226 \mathrm{~mL}$, median hospital stay was 11.2 days. 1 patient had Clavien-Dindo grade 3 complication in the form of intra-abdominal collection requiring percutaneous drain placement. Six patients had significant elevation of transaminases that resolved by a median duration of 8 days. No patient had post-hepatectomy liver failure. None of the patients developed recurrent hepaticolithiasis or biliary stricture, with follow-up duration of 11 to 64 months.

Conclusions: Major hepatic resection is a safe and recommended procedure for hepaticolithiasis, especially in presence of hepatic lobar atrophy. It carries acceptable morbidity and good outcomes when performed by an experienced hepatobiliary surgical team. A tertiary care centre with good imaging and liver volumetry facilities is best suited for this line of management. 